\title{
Small interfering RNA-producing loci in the ancient parasitic eukaryote Trypanosoma brucei
}

\author{
Christian Tschudi ${ }^{1}$, Huafang Shi ${ }^{2}$, Joseph B Franklin ${ }^{3}$ and Elisabetta Ullu ${ }^{2,3^{*}}$
}

\begin{abstract}
Background: At the core of the RNA interference (RNAi) pathway in Trypanosoma brucei is a single Argonaute protein, TbAGO1, with an established role in controlling retroposon and repeat transcripts. Recent evidence from higher eukaryotes suggests that a variety of genomic sequences with the potential to produce double-stranded RNA are sources for small interfering RNAs (siRNAs).

Results: To test whether such endogenous siRNAs are present in T. brucei and to probe the individual role of the two Dicer-like enzymes, we affinity purified TbAGO1 from wild-type procyclic trypanosomes, as well as from cells deficient in the cytoplasmic (TbDCL1) or nuclear (TbDCL2) Dicer, and subjected the bound RNAs to Illumina high-throughput sequencing. In wild-type cells the majority of reads originated from two classes of retroposons. We also considerably expanded the repertoire of trypanosome siRNAs to encompass a family of 147-bp satellite-like repeats, many of the regions where RNA polymerase II transcription converges, large inverted repeats and two pseudogenes. Production of these newly described siRNAs is strictly dependent on the nuclear DCL2. Notably, our data indicate that putative centromeric regions, excluding the CIR147 repeats, are not a significant source for endogenous siRNAs.
\end{abstract}

Conclusions: Our data suggest that endogenous RNAi targets may be as evolutionarily old as the mechanism itself.

Keywords: Argonaute, Trypanosome, Dicer-deficient, Retrotransposon, Inverted repeat, Convergent transcription unit, siRNA

\section{Background}

The "natural" or endogenous RNA interference (RNAi) pathway functions as a genome immune defense mechanism to maintain genome integrity, prevents viral infection and limits the potential deleterious consequences of transposon/retroposon mobilization [1-3]. Whereas deep sequencing of endogenous small interfering RNAs (siRNAs) has confirmed that the large majority of siRNAs in several model organisms, including insects [4], plants [5] and mammals [6,7], are indeed derived from retroposons and transposons, these studies also uncovered new classes of small RNAs originating among others from regions of the genome, where convergent transcription occurs or from loci predicted to generate double-stranded RNA (dsRNA)

\footnotetext{
* Correspondence: elisabetta.ullu@yale.edu

${ }^{2}$ Department of Internal Medicine, School of Medicine, Yale University, New Haven, CT 06536, USA

${ }^{3}$ Department of Cell Biology, School of Medicine, Yale University, New Haven, CT 06536, USA

Full list of author information is available at the end of the article
}

by a fold back mechanism, i.e. inverted repeats. In addition, tRNA- and snoRNA-derived RNA fragments have recently been added to the catalogue of small RNAs implicated in RNAi-related gene silencing pathways [8-12].

The processing of dsRNA is executed by a member of the Dicer family of RNase III-related enzymes to yield a variety of 21-30 nt small dsRNAs that are then loaded into a specific class of Argonaute (AGO)-family proteins $[13,14]$. RNAi was first described in the parasitic protozoan Trypanosoma brucei in 1998 [15] and to date we know that there are two distinct Dicer-like enzymes in these organisms, namely TbDCL1 [16] and TbDCL2 [17], which are mostly present in the cytoplasm and in the nucleus, respectively. siRNAs generated by both Dicers are transferred to TbAGO1, the sole slicer responsible for cleavage of target transcripts $[18,19]$ with the assistance of TbRIF4, a 3'-5' exonuclease [20].

Soon after the discovery of RNAi in T. brucei, "oldfashioned" sequencing of an 20-30 nt RNA library revealed that the two main classes of retroposons,

\section{Biomed Central}


namely ingi and SLACS, were sources of siRNAs [21] and subsequent sequencing of siRNAs derived from TbAGO1 immunoprecipitates uncovered a new class of siRNAs from 147-bp tandem units [17], which we named CIR147 repeats (Chromosome Internal Repeats). These satellite-like repeats are located in non-telomeric regions of $T$. brucei chromosomes 4, 5, and 8 and were independently identified as part of putative centromeric regions [22], although at present we cannot exclude the possibility that these repeats also exist on the other chromosomes. In addition, functional studies were consistent with a major role for endogenous RNAi in T. brucei to maintain genome integrity $[17,23]$. On the other hand, the unique organization of the trypanosome genome into long directional gene clusters with many sites of convergent transcription [24] raised the possibility of additional sources of siRNAs and thus may be pointing to new role(s) for RNAi in these organisms. Thus, using deep sequencing we surveyed the small RNAs associated with Argonaute 1 in wild-type cells, as well as in cells devoid of either one of the two Dicers to gauge their respective role in small RNA production.

\section{Results}

\section{Small RNA data sets}

To provide a comprehensive catalogue of small RNAgenerating loci in the procyclic $T$. brucei YTat 1.1 strain [25], we subjected RNAs associated with TbAGO1 to next-generation sequencing on the Illumina platform and the distribution of siRNAs along the T. brucei 11 mega chromosomes (GeneDB version 5) was analyzed using a bioinformatics pipeline described in Methods. In addition to wild-type cells, we surveyed small RNAs in cells lacking either TbDCL1 or TbDCL2, to gain further insight into the distinct role of the two Dicers in the RNAi pathway. Alignment to the reference T. brucei brucei TREU 927 genome [24] with 93\% identity yielded $8,775,792,7,821,630$ and 4,722,271 total reads (Table 1), representing 842,296, 744,681 and 545,597 unique sequences (Additional file 1) for the wild-type, $\mathrm{dcl1}^{-/-}$and $d c l 2^{-/-}$libraries, respectively (The sequence data from this study have been submitted to the NCBI Sequence Read Archive - SRA at http://www.ncbi.nlm.nih.gov/Traces/sra/ sra.cgi - under accession no. SRA057341). The overall

Table 1 Summary of total small RNA reads

\begin{tabular}{llll}
\hline & wt & $\boldsymbol{d} \mathbf{c l} \mathbf{1}^{-/-}$ & $\boldsymbol{d c l \mathbf { 2 } ^ { - / }}$ \\
\hline Total reads & $10,001,135$ & $9,199,808$ & $5,829,677$ \\
Reads mapped to the & & & \\
11 mega chromosomes & $8,775,792$ & $7,821,630$ & $4,722,271$ \\
Annotated reads & $6,826,892$ & $5,987,935$ & $3,400,947$ \\
Unassinged reads & $1,948,900$ & $1,833,695$ & $1,321,324$ \\
Unmapped reads & $1,225,343$ & $1,378,178$ & $1,107,406$ \\
\hline
\end{tabular}

distribution of reads did not change noticeably, if the alignment was performed by increasing the \% identity to $95 \%$ or $100 \%$, albeit the read density was reduced uniformly.

To categorize small RNA-producing loci, we inspected the aligned data for regions that corresponded to annotated features. In addition, we surveyed the $T$. bruce $i$ genome for tandem and inverted repeats, which to our knowledge has not been done systematically, and asked whether they are sources of small RNAs. This strategy allowed us to annotate $6,826,892$ of the reads (78\%) in the wild-type library and establish seven categories of bona fide siRNA sources (Table 2), which were analyzed further as described below. The reads not matching to the 11 mega chromosomes $(1,225,343$ or $12 \%)$ might arise from unsequenced regions of the genome or from sequencing errors. Indeed, an additional 638,046 reads aligned to the $T$. brucei gambiense genome, a closely related trypanosomatid, with no genomic regions rising above background levels (data not shown). In addition, de novo assembly of the remaining reads did not generate contigs that would point to additional genuine siRNA-producing loci (data not shown).

The small RNAs recognized as siRNAs displayed a distinct size distribution ranging in size from 22 to 25 nucleotides (Figure 1, wild-type), which mirrors our previous estimates $[17,21,23]$. siRNAs isolated from cells deficient in TbDCL2 were slightly larger (Figure 1 and see below), in line with our previous observation that siRNAs processed in an in vitro extract from $d c l 2^{-/-}$cells were one nucleotide longer than the wild-type size [17]. In contrast, reads aligning to rRNAs $(97,847$ total reads or $1.4 \%)$ and tRNAs/snoRNAs (9,327 total reads or $0.1 \%)$, did not display a length distribution characteristic of siRNAs, but had a broad distribution between 18 and 32 nucleotides (data not shown). Furthermore, we

Table 2 Summary of small RNA classes in procyclic T. brucei

\begin{tabular}{|c|c|c|c|}
\hline & wt & $d c / 1^{-/-}$ & $d c / 2^{-/-}$ \\
\hline ingi/RHS & $3,555,719(52.1 \%)$ & $3,151,779(52.6 \%)$ & $2,380,772(70.0 \%)$ \\
\hline SLACS & $1,793,319$ (26.3\%) & $1,546,465$ (25.8\%) & $486,558(14.3 \%)$ \\
\hline CIR147 & 586,988 (8.6\%) & 339,312 (5.7\%) & $4,953(0.2 \%)$ \\
\hline $\mathbb{I R}^{\mathrm{a}}$ & $452,056(6.6 \%)$ & $282,956(4.7 \%)$ & 105,792 (3.1\%) \\
\hline$C T U^{b}$ & $179,694(2.6 \%)$ & 265,799 (4.4\%) & 151,045 (4.4\%) \\
\hline Pseudogenes & $55,460(0.8 \%)$ & 45,079 (0.8\%) & 179 (0.01\%) \\
\hline Miscellaneous $^{c}$ & 92,099 (1.4\%) & 70,815 (1.2\%) & $4,169(0.11 \%)$ \\
\hline rRNAs & 97,847 (1.4\%) & $265,895(4.4 \%)$ & $253,779(7.5 \%)$ \\
\hline t/snoRNAs ${ }^{d}$ & $9,327(0.1 \%)$ & $18,574(0.3 \%)$ & $13,272(0.4 \%)$ \\
\hline
\end{tabular}




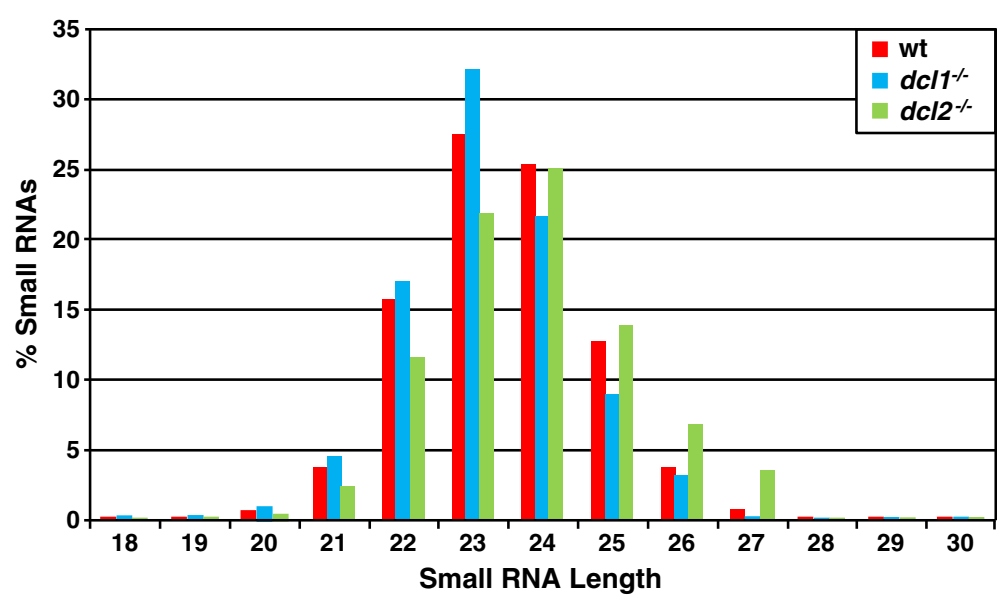

Figure 1 Size distribution of analyzed $T$. brucei small RNAs in wild-type (wt), $d c l 1^{-/-}$and $d c / 2^{-/-}$cells.

carefully inspected reads originating from tRNAs and snoRNAs, since recent reports have highlighted a novel class of small RNAs originating from these structural RNAs [8-12]. Our analysis revealed that reads aligning to trypanosome tRNAs and snoRNAs had a random distribution and did not suggest cleavage in a specific manner, reminiscent of tRNA- or snoRNA-derived small RNAs implicated in RNA silencing, Thus we classified them as degradation products. Finally, we did not find evidence for the presence of micro RNAs in the libraries we generated from the procyclic T. brucei YTat 1.1 strain.

\section{The retroposons ingi and SLACS are major sources for small RNAs}

Consistent with our earlier results [21], the retroposons ingi and SLACS were by far the two major sources of small RNAs accounting for $78 \%$ of all reads in the wildtype library (Table 2). Ingi is part of a group of related, but rather heterogeneous retroposon-like sequences present throughout the genome, which also includes the ribosomal inserted mobile elements (RIME) and the retrotransposon hot spot (RHS) family [26,27]. In our analysis we pooled all the reads aligning to these various elements and thus in the wild-type library this group of retroposons was responsible for $52 \%$ of the siRNAs (Table 2). The "LINE-like" ingi is a $5.25 \mathrm{~kb}$ element composed of an ingi-specific $4.75 \mathrm{~kb}$ fragment flanked by two separate halves of the RIME element family $[28,29]$. A potentially functional retroposon contains a single long ORF (4,971 bp), which encodes a 1,657 amino acid protein with a predicted reverse transcriptase and endonuclease domain (Figure 2A and refs. [30,31]). We used such an element to gauge the distribution of siRNAs, which turned out to be fairly even along the entire retroposon with no obvious gaps and with a similar distribution of reads coming from the sense or antisense strand (Figure 2A).
SLACS, or Spliced Leader Associated Conserved Sequence, is a site-specific non-LTR retroposon that integrates exclusively into the spliced leader (SL) RNA gene [32]. Our wild-type Ytat 1.1 strain, which was used for the small RNA sequencing described here, has between 16 to 18 copies of the SLACS element per haploid genome (ref. [33] and data not shown). The element is $6.8 \mathrm{~kb}$ long and the two predicted ORFs encode a putative reverse transcriptase and endonuclease domain (Figure 2B). In the wild-type library $26 \%$ of the total reads ( $16 \%$ of the unique reads) aligned to this retroposon with approximately twice as many reads originating from the antisense strand. However, in contrast to ingi, siRNAs were restricted to the $5^{\prime}$ half of the element with very few reads in the 3 ' half (Figure $2 \mathrm{~B}$ ). Consistent with our analysis of siRNA abundances in TbDCL1 and TbDCL2 KO cells by Northern blots [17], siRNAs numbers in cells lacking TbDCL1 were slightly lower, but were reduced to $14 \%$ in TbDCL2 KO cells (Table 2). The uneven distribution of siRNAs was maintained in both KO libraries.

\section{Small RNAs originate from CIR147 repeats, but not from other tandem repeats}

In the wild-type library $8.6 \%$ of the total reads $(586,988$ reads) aligned to the CIR147 tandem repeats (Table 2). The abundance of CIR147 siRNAs was reduced slightly to $5.7 \%$ in the absence of TbDCL1, whereas in the DCL2KO library only $0.2 \%$ of the siRNAs originated from these repeats, thus confirming our functional studies that TbDCL2 has a primary role in the generation of siRNAs from CIR147 repeats [17].

CIR147 repeats on chromosomes 4, 5 and 8 were previously identified as part of putative $T$. brucei centromeric regions based on etoposide-mediated topoisomerase-II cleavage [22]. Since centromeres in the fission yeast Schizosaccharomyces pombe are under the control of 


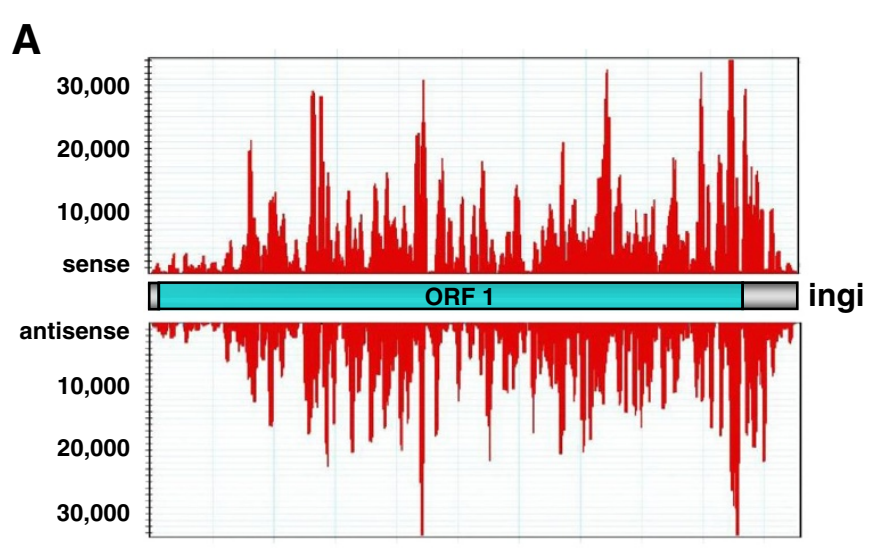

B

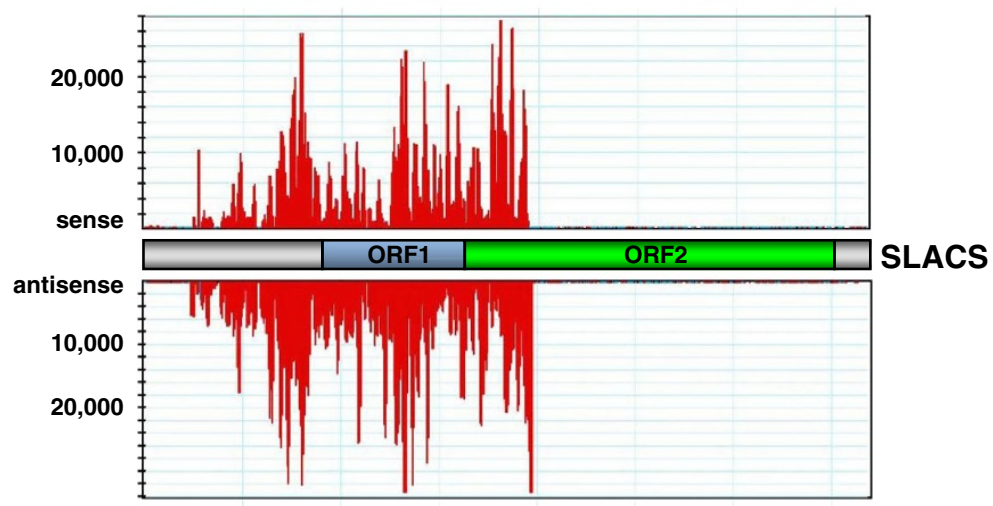

Figure 2 Distribution of small RNAs aligning to retroposon elements. (A) ingi is a $5.25 \mathrm{~kb}$ element composed of an ingi-specific $4.75 \mathrm{~kb}$ fragment flanked by two separate halves of the RIME element family [28,29]. This element contains a single ORF (ORF1) flanked by two RIME elements. 2,326,864 reads in the wild-type library aligned to this element with 1,1321,119 reads from the sense and 1,194,745 reads from the antisense strand. Note that sense and antisense small RNA reads are shown separately. (B) The SLACS element is 6.8 kb long with two predicted ORFs. Sense (640,935 reads) and antisense (1,152,384 reads) small RNA reads are shown separately.

the RNAi pathway [34], we surveyed other predicted centromeric regions in T. brucei for the production of small RNAs. However, none of the putative centromeric regions on chromosomes 1, 2, 3, 6 and 7 [22] showed a significant accumulation of reads as compared to flanking regions (data not shown). Of note is that putative centromeres were not mapped on the largest three chromosomes, i.e. 9, 10 and 11 [22]. The lack of reads at the putative centromeres on chromosomes 2, 3 and 7 was particularly intriguing, since they contain large arrays of tandem repeats of 29, 120 and 59 base pairs, respectively (ref. [22] and data not shown), suggesting that short tandem repeats per se are not destined to be a source for small RNAs in procyclic $T$. brucei. To test this hypothesis, we used the program tandem repeats finder [35], tabulated all repeat arrays on the 11 mega base chromosomes (data not shown) and then manually inspected repeats with a size $>10 \mathrm{bp}$ and a copy number $>10$ for aligned reads. Although many additional tandem repeats were identified, none was found to be a source of small RNAs (data not shown). Thus, it appeared from this analysis that the CIR147 repeats are the only tandem repeats in the T. brucei genome generating siRNAs and that putative centromeric regions not harboring CIR147 repeats are devoid of siRNAs.

\section{New loci for small RNA generation: pseudogenes and inverted repeats}

Once we recognized all the siRNAs originating from retroposons and CIR147 repeats, we were left with 13\% (890,866 reads) of the reads aligning to potential small RNA-producing loci in the T. brucei genome. Based on similar studies in Drosophila and mammals [6,36,37], we considered additional possible sources of small RNAs, i.e. pseudogenes and inverted repeats. In the GeneDB release 5 of the T. brucei genome [24], which was used in our analysis, there are 1,279 annotated pseudogenes with the majority assigned to variant surface glycoprotein (VSG) genes and expression-site-associated genes (ESAG), as well as a few for other protein coding genes. In the library from wild-type procyclic cells there was no apparent increased accumulation of reads at VSG or ESAG 
pseudogenes, as compared to flanking regions, but there were two regions, currently annotated as pseudogenes but for which the corresponding "genes" have not yet been identified, that generated small RNAs, namely Tb927.5.290 (51,758 total reads) and Tb09.160.3400 (3,702 total reads). Small RNAs from these two loci appeared to be predominantly generated by $T b D C L 2$, since ablation of TbDCL1 barely affected the accumulation of siRNAs, whereas the lack of TbDCL2 reduced the small RNA levels to $5 \%$ or lower relative to wild-type (Table 2, Additional file 4 and Figure 3).

To the best of our knowledge, the current $T$. brucei genome has not been surveyed for the presence of large inverted repeats (IR). Thus, we used the program inverted repeat finder [38], as well as BLAST [39] to align each chromosome with itself, and identified 7 inverted repeats ranging in size from 422 bp to 10,011 bp (Table 3). Remarkably, at the sequence level each repeat pair is essentially identical with only 14 mismatches for the longest one. To ascertain that these were bona fide inverted repeats and not genome assembly artifacts, we verified the presence of IR3 (3,636 bp) and IR4 (2,520 bp) in our YTat 1.1 strain by PCR (data not shown).

Inverted repeats accounted for $6.6 \%$ of small RNAs in the wild-type library, with the number of reads per inverted repeat varying from 403 for IR1 to 372,045 for IR3 (Table 3). Curiously and similar to what we noticed with the SLACS retroposon (Figure 2B), the small RNAs
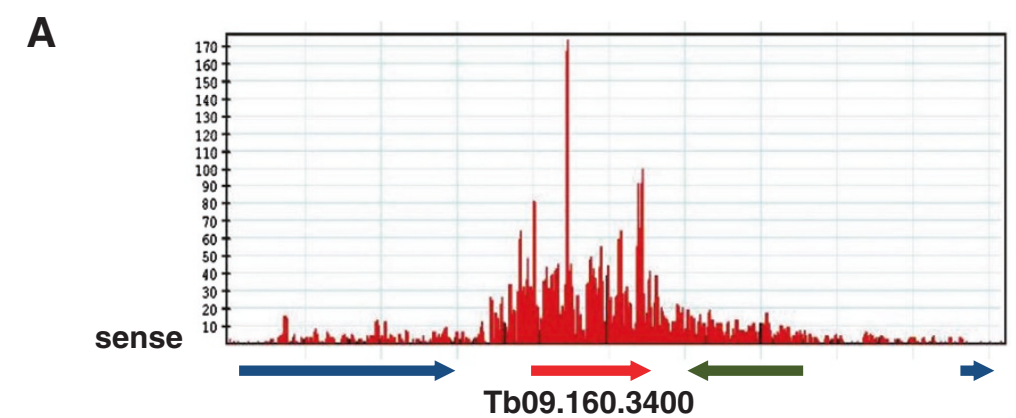

antisense

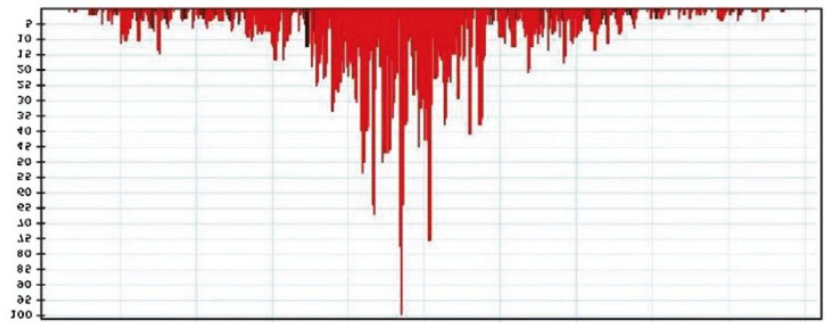

B

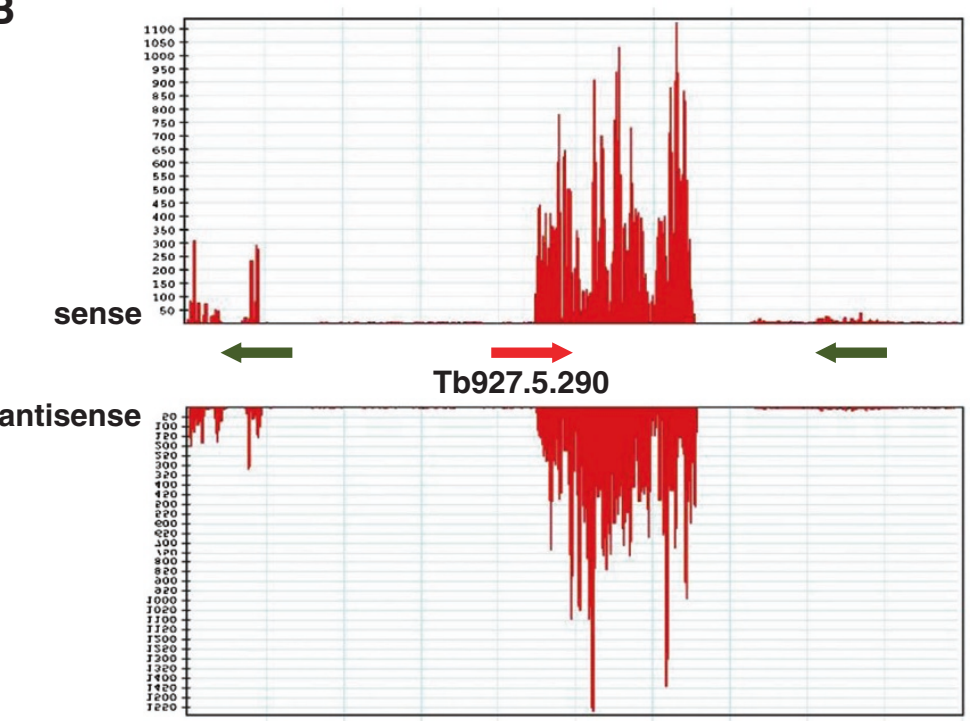

Figure 3 Small RNAs originating from two annotated pseudogenes, namely Tb09.160.3400 (A) and Tb927.5.290 (B). Sense and antisense reads are shown. 
Table 3 Small RNAs at long inverted repeats in T. brucei

\begin{tabular}{|c|c|c|c|c|c|c|}
\hline Name & Start/End & $\begin{array}{l}\mathrm{RL}^{\mathrm{a}} \\
\text { (bp) }\end{array}$ & Identity & $\begin{array}{l}\text { wt } \\
\text { total reads }\end{array}$ & $\begin{array}{l}d c / 1 \\
\% w t\end{array}$ & $\begin{array}{l}d c / 2 \\
\% w t\end{array}$ \\
\hline Tb927_02_IR1 & $898600 / 902200$ & 422 & $100 \%$ & 403 & $52 \%$ & $9 \%$ \\
\hline Tb927_02_IR2 & $994300 / 100060$ & 10,011 & $99 \%\left(14^{b}\right)$ & 8,681 & $56 \%$ & $24 \%$ \\
\hline Tb927_07_IR3 & $1125000 / 1134900$ & 3,636 & $99 \%(1)$ & 372,045 & $73 \%$ & $41 \%$ \\
\hline Tb927_07_IR4 & 1889100/1896500 & 2,520 & $100 \%$ & 55,854 & $71 \%$ & $103 \%$ \\
\hline Tb927_08_IR5 & $1048400 / 1059500$ & 1,743 & $99 \%(5)$ & 1,390 & $108 \%$ & $49 \%$ \\
\hline Tb927_10_IR6 & $467000 / 478000$ & 853 & $99 \%(8)$ & 4,387 & $38 \%$ & $6 \%$ \\
\hline Tb927_11_IR7 & $4219000 / 4231000$ & 1,814 & $99 \%(3)$ & 9,296 & $52 \%$ & $2 \%$ \\
\hline
\end{tabular}

${ }^{\mathrm{a}} \mathrm{RL}$, repeat length.

${ }^{b}$ number of mismatches between the two repeats.

originated from restricted regions of the inverted repeat and did not cover the entire length (Figure 4A). In addition, the region separating the two repeats was also a source of small RNAs, suggesting that transcription occurred on both strands. In all cases but one (IR5), the read count was reduced to between $39 \%$ and $75 \%$ of wild-type levels in the absence of TbDCL1. The involvement of $\mathrm{TbDCl} 2$ in the generation of small RNAs from inverted repeats varied, with small RNAs derived from IR1, IR6 and IR7 clearly dependent on the nuclear Dicer, whereas IR2, IR3 and IR5 appeared to be less dependent (Table 3). Finally, the generation of small RNAs originating from IR4 appeared to be only slightly dependent on either Dicer by the sequencing analysis. The generation of small RNAs from inverted repeats and their dependence on the two Dicers was validated by Northern blotting for IR3 (Figure 4B).

\section{Convergent transcription units are a source of small RNAs}

One peculiar feature of the T. brucei genome is the organization of protein coding genes into long directional clusters [24]. A corollary of this trait is that in chromosomal regions were units converge, there is the potential for overlapping (bi-directional) transcription, which could potentially lead to the formation of dsRNA and subsequent small RNAs. We surveyed 49 convergent
A

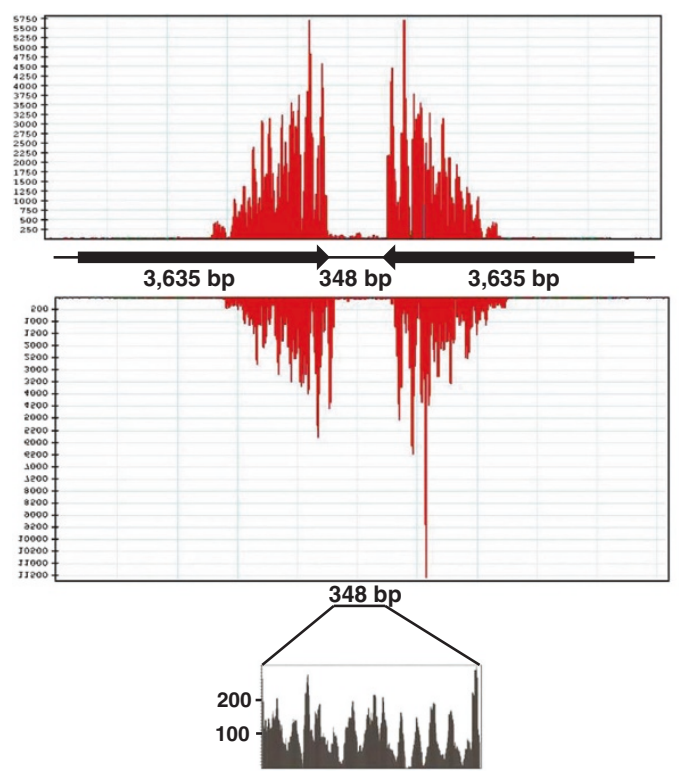

B

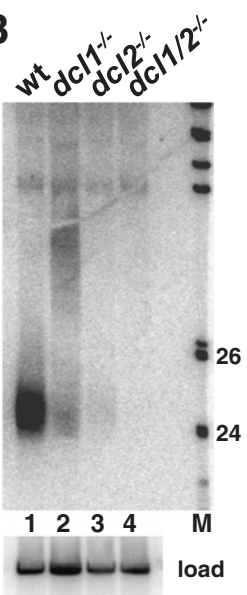

Figure 4 Long inverted repeats are a source of small RNAs. (A) Small RNA distribution on IR3. Reads were distributed randomly on the two repeats during alignment. An enlargement of the region in-between the two repeats is shown below. (B) Low-molecular weight RNAs isolated from various cell lines as indicated above each lane were separated by denaturing gel electrophoresis and analyzed by Northern hybridization with an IR3-specific probe. Hybridization to 5 S rRNA was used as a loading control (load, bottom panel). See additional file 5 for sequences of the hybridization probes. 
transcription units (CTUs) on the 11 megabase chromosomes and in each case we detected significant accumulation of reads in these regions, as compared to flanking sequences (Additional file 2). For the majority of CTUs accumulation of small RNAs was not dependent on TbDCL1, except for CTU4, CTU17, CTU40 and CTU41, where ablation of $T b D C L 1$ reduced the read count to about $60 \%$ of wild-type levels. In contrast, small RNA accumulation at all CTUs was critically dependent on TbDCL2. We validated the production of small RNAs at one of the convergent units (CTU50) by Northern blot analysis, which also corroborated the involvement of $T b \mathrm{Dcl} 2$ in the formation of small RNAs (Figure 5).

The production of siRNAs at convergent transcription units raises the question whether these siRNAs modulate gene expression, since they overlap with annotated genes. We chose two CTUs (CTU36 and CTU48) and monitored the steady-state levels of two mRNAs in each unit in RNA isolated from wild-type and $\mathrm{dcl}^{-/-}$cells by Northern blot analysis (Figure 6). In both CTUs there was no detectable change in the accumulation of mRNAs in the absence of $T b D C L 2$, thus questioning the contribution to gene expression of siRNAs generated in convergent transcription units.

\section{Discussion}

Given the wide distribution of RNAi and related phenomena in all branches of the eukaryotic lineage, a case can be made that the RNAi mechanism originated early during eukaryotic evolution and, as an extension, the repertoire of small RNAs generated by the RNAi pathway should have an evolutionary history. To explore this hypothesis, we have been studying the mechanism and biological function of RNAi in the ancient parasitic protozoan T. brucei. This lead to the realization several years ago that the two retroposons in the genome, namely ingi and SLACS, were a source of siRNAs, thus implicating RNAi in maintaining genome integrity [21]. The next observation we made was that a subclass of putative centromeres containing 147-bp tandem repeats were also generating siRNAs [17]. In the current study we aimed to annotate all small RNA-producing loci in insect-form derived trypanosomes by deep-sequencing RNAs associated with the sole Argonaute 1 slicer. In addition, to further our understanding of the specific role of the two Dicers in this organism, we surveyed small RNAs in cells deficient in either the nuclear or cytoplasmic Dicer.

Our results exposed a number of intriguing features and thus raise numerous questions. Firstly, siRNAs originating from SLACS were restricted to the 5' half of the element (Figure 2B), suggesting a possible avenue for the generation of double-stranded RNA. Our studies on the expression of the SLACS element suggested that transcription initiates at the +1 position of the interrupted spliced leader RNA gene and continues through the 5' UTR and ORF1 [33]. In addition, preliminary experiments indicated a detectable amount of antisense transcription, although the low level of transcription made it impossible to pinpoint the extent of transcription, let alone the site of transcription initiation [33]. Nevertheless, having a defined landmark from our read alignments, it
A

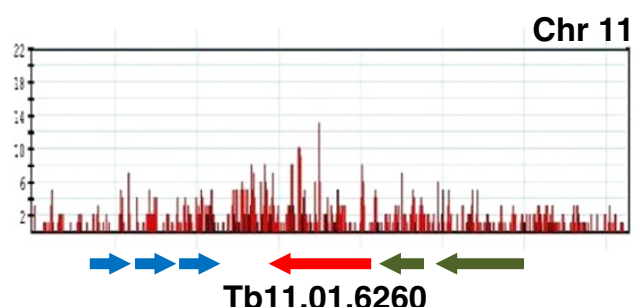

Tb11.01.6260

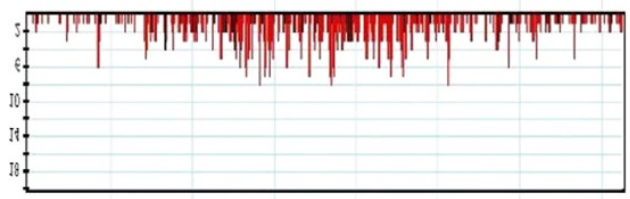

B

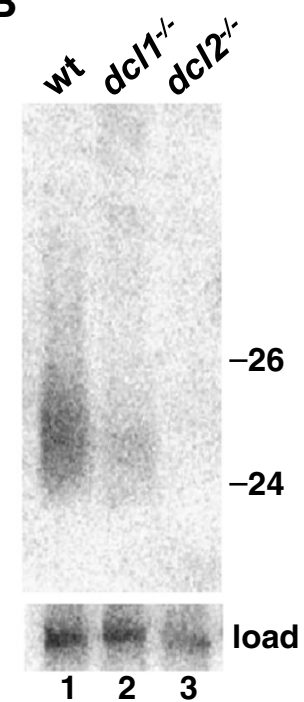

Figure 5 Validation of small RNA accumulation at CTU50. (A) Small RNA distribution. (B) Low-molecular weight RNAs isolated from various cell lines as indicated above each lane were separated by denaturing gel electrophoresis and analyzed by Northern hybridization with two oligonucleotides representing the most abundant reads. Loading control (load), hybridization to 5 S rRNA. See additional file 5 for sequences of the hybridization probes. 

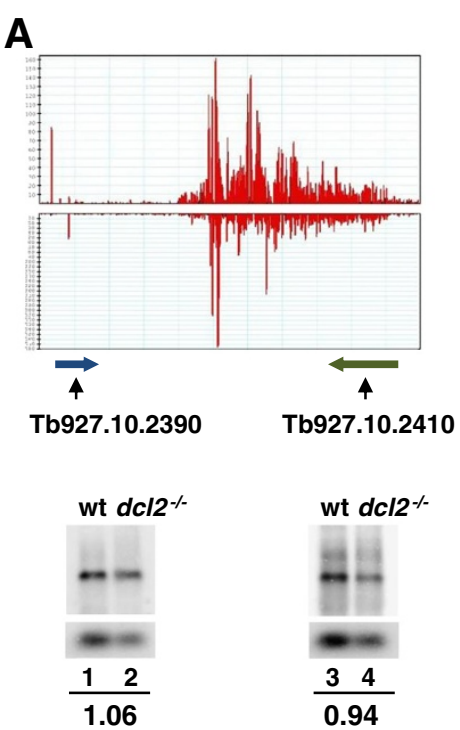

B
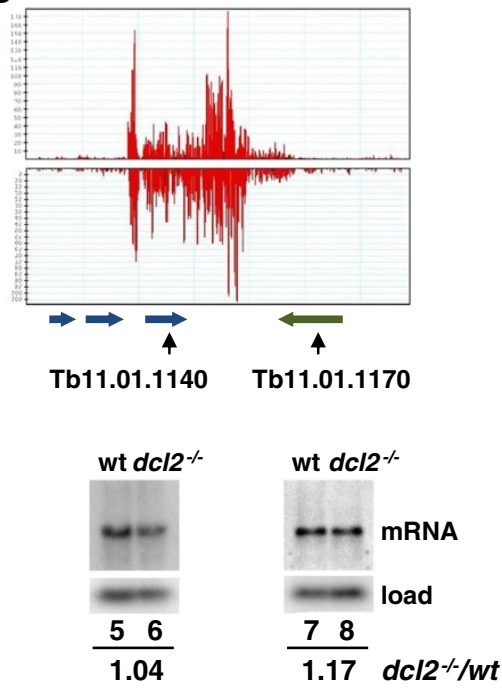

Figure 6 Steady-state mRNA levels at two convergent transcription units in wild-type and DCL2KO cells. (A) and (B) Total RNA was prepared from wild-type and $\mathrm{dCl}^{-2_{-}}$cells and analyzed by Northern hybridization with a probe specific for the genes indicated below the read distribution. See additional file 3 for sequences of the hybridization probes. The hybridization was quantitated by Phosphorlmager analysis, normalized to the loading control (tRNA) and expressed as a ratio between $d c / 2^{-/-}$and wt.

might be possible in future experiments to delineate the origin of the double-stranded RNA.

Secondly, the CIR147 repeats present in putative centromeric regions on three chromosomes were the only tandem repeats in the genome giving rise to small RNAs, despite the presence of other short tandem repeats either in putative centromeres or other genomic regions. What is so special about the CIR147 repeats that they are under the control of the RNAi pathway and how are siRNAs, i.e. dsRNA, produced from these arrays? At present there is no knowledge of what constitutes a centromere in trypanosomes and whether heterochromatin is crucial for centromere function. More importantly, a very basic question to put forward is whether the silent transcriptional status of the CIR147 repeats in wild-type cells [17] is caused by a heterochromatic state of the locus. In Drosophila and in yeast, $\mathrm{H} 2 \mathrm{AZ}$ was reported to be involved in heterochromatic silencing $[40,41]$. This histone variant has been characterized in $T$. brucei and shown to function in concert with a novel H2B variant, H2BV [42]. By ChIP, both proteins were shown to be associated with highly repetitive DNA, including the mini-chromosomal 177-bp repeats, the expression site-proximal 50-bp repeats, and telomeric repeats. Intriguingly, $\mathrm{H} 2 \mathrm{AZ}$ and $\mathrm{H} 2 \mathrm{BV}$ do not co-localize with sites of nascent RNA transcription, suggesting that they are primarily enriched at transcriptionally inactive regions of the genome. Given our data, it is tempting to speculate that these two histone variants might be involved in the regulation of the transcriptional status of the 147-bp repeat clusters, a possibility we are currently investigating.
Taking advantage of RNAi-deficient cells, we know that both strands of the CIR147 repeats generate transcripts of over $10 \mathrm{~kb}$ and based on $\alpha$-amanitin sensitivity are synthesized by RNA polymerase II [17]. Although the latter observation might be in line with studies in other organisms, where it has been shown that RNA polymerase II appears to play a direct role in heterochromatin assembly [43], the assembly of specialized chromatin domains in T. brucei and a possible connection with RNAi remains shrouded in mystery.

Thirdly, we identified 7 large inverted repeats in the T. brucei genome and all generated small RNAs, albeit to different levels. In itself these long inverted repeats are a curiosity, since in many organisms such structures can have a serious effect on genome stability [44]. In particular, the remarkably high sequence identity suggests some selective pressure maybe relying on the formation of a hairpin structure at the DNA or RNA level. It is tempting to speculate that RNAi might play a role in maintaining these repeats.

Fourthly, our data highlighted two annotated pseudogenes that are a source of small RNAs, whereas the majority of pseudogenes, i.e. VSGs and ESAGs, do not generate small RNAs in procyclic trypanosomes. In addition, TbDCL2, and not TbDCL1, appeared to be mainly responsible for these pseudogene-derived small RNAs. Our results seem to be in contrast with a recent study in bloodstream-form trypanosomes, where small RNAs originating mainly from VSG and RHS pseudogenes were found to be dependent on TbDCL1 [45]. However, since we have noted differences in the contribution of the two 
dicers in the generation of small RNAs from inverted repeats (Table 3), one cannot exclude a similar scenario for pseudogene-derived small RNAs, especially considering that the two studies were done in different life-cycle stages.

Lastly, we detected siRNAs at all convergent transcription units, although the distribution of the small RNAs varied greatly. At present we can only speculate about the functional significance for the existence of siRNAs originating from CTUs. Our analysis of steady-state mRNA levels at two selected CTUs (Figure 6) in wild-type and DCL2KO cells would indicate that RNAi does not play a role in the modulation of mRNA levels at these loci in procyclic cells. However, it is possible that the number of siRNAs originating from CTUs, namely 8,088 and 7,394 for the two we tested, is too low to have a direct effect on gene expression. Alternatively, siRNAs from CTUs could induce DNA or chromatin modifications at the homologous genomic locus. Another open question is the origin of these siRNAs, i.e. how is the dsRNA generated in the absence of evidence that the converging transcription units overlap. At present it is not known how and where transcription terminates at CTUs, although the presence of siRNAs at CTUs might suggest that transcription proceeds to some extent into the adjacent gene cluster. In support of this hypothesis our recent transcriptome studies using RNA-Seq [46] revealed a low level of anti-sense transcription at CTUs (unpublished observation), providing a possible avenue for the formation of dsRNA. Experiments are ongoing to corroborate this scenario and to investigate the uneven distribution of small RNAs among CTUs.

\section{Conclusions}

The results presented here significantly expanded our earlier sequencing and functional studies that retroposonand CIR147 repeat-derived siRNAs represent the major sources of small RNAs and expanded the repertoire to include small RNAs originating from inverted repeats, pseudogenes and loci of converging transcription units. At the same time, our data set derived from procyclic form trypanosomes did not reveal the presence of miRNAs, as well as tRNA- or snoRNA-derived RNA fragments generated by the RNAi pathway. However, this conclusion does not rule out the possibility that other stages of the trypanosome life cycle might generate a different set of small RNAs.

Our data revealed a dominant role for the nuclear TbDCL2 in the endogenous T. brucei RNAi pathway and the landscape of siRNAs in this ancient eukaryotic parasite closely mirrors that described in metazoans, such as Drosophila and mouse, attesting to the early evolutionary origin of RNAi.

\section{Methods}

\section{Standard methods}

Previously published procedures were followed for culturing trypanosome YTat 1.1 cells [15], generation of knockout cell lines by PCR-based methods [47] and Northern blots of total RNA [21]. Oligonucleotides used to prepare probes for Northern blots are listed in Additional file 5.

\section{Small RNA library preparation, sequencing and read processing}

TAP-tagged TbAGO1 was expressed in wild-type cells [23], TbDCL1KO and TbDCL2KO cells (this study) and following TbAGO1 affinity purification, the bound small RNAs were prepared for sequencing. For the library construction we essentially followed the protocol suggested by the manufacturer (http://www.illumina.com/).

Libraries were sequenced on an Illumina GAII platform at the Yale Center for Genome Analysis and the reads of $35 \mathrm{nt}$ in length were pre-processed using the FASTX-toolkit on the public Galaxy webserver ([48-50]; http://galaxyproject.org/). Following removal of the adaptor sequences, reads were collapsed to non-redundant datasets and short reads $(<=17 \mathrm{bp})$ and low complexity reads (including poly $\mathrm{A} / \mathrm{C} / \mathrm{G} /$ or $\mathrm{T}$, di-nucleotide repeats, etc.) were removed. We mapped processed reads, both total and non-redundant reads, to the T. brucei 11 mega chromosomes (GeneDB version 5) using Bowtie [51] and the Lasergene 9.1 software package from DNASTAR (http://www. dnastar.com/).

\section{Additional files}

Additional files 1: Summary of total and unique reads.

Additional files 2: Total and unique siRNAs at two pseudogenes in wild-type (wt), $\boldsymbol{d c l} \mathbf{1}^{-/-}$and $\boldsymbol{d} \mathbf{c l} \mathbf{2}^{-1-}$ cells. siRNAs were normalized to the total number of reads (setlength).

Additional files 3: Total small RNAs at each convergent transcription units (CTUs) in wild-type (wt), $d \mathrm{cl}^{-/-}$and $d \mathrm{cl}^{-/-}$cells. siRNAs were normalized to the total number of reads (setlength).

Additional files 4: Sequences of oligonucleotides used for preparing probes for hybridization.

Additional files 5: Total small RNAs at various regions of the genome with no clear features. Listed as "miscalleneous" in Table 2.

\section{Competing interests}

The authors declare that they have no competing interests.

\section{Authors' contributions}

$C T$ participated in the design of the study, analyzed the data and drafted the manuscript. HS performed the molecular studies. JBF participated in the bioinformatics analysis. EU conceived the study, participated in its design and coordination and edited the manuscript. All authors read and approved the final manuscript.

\section{Acknowledgements}

This work was supported by Public Health Service grants Al28798 and Al56333 to E.U. 


\section{Author details}

'Department of Epidemiology of Microbial Diseases, School of Public Health, Yale University, New Haven, CT 06536, USA. ²Department of Internal Medicine, School of Medicine, Yale University, New Haven, CT 06536, USA

${ }^{3}$ Department of Cell Biology, School of Medicine, Yale University, New Haven, CT 06536, USA.

Received: 25 March 2012 Accepted: 24 August 2012

Published: 27 August 2012

\section{References}

1. Plasterk RH: RNA silencing: the genome's immune system. Science 2002, 296:1263-1265.

2. Girard A, Hannon GJ: Conserved themes in small-RNA-mediated transposon control. Trends Cell Biol 2008, 18:136-148.

3. Ketting RF: The many faces of RNAi. Dev Cell 2011, 20:148-161.

4. Okamura K, Chung WJ, Ruby JG, Guo H, Bartel DP, Lai EC: The Drosophila hairpin RNA pathway generates endogenous short interfering RNAs. Nature 2008, 453:803-806.

5. Rajagopalan R, Vaucheret $H$, Trejo J, Bartel DP: A diverse and evolutionarily fluid set of microRNAs in Arabidopsis thaliana. Genes Dev 2006, 20: 3407-3425.

6. Watanabe T, Totoki Y, Toyoda A, Kaneda M, Kuramochi-Miyagawa S, Obata Y, Chiba H, Kohara Y, Kono T, Nakano T, et al: Endogenous siRNAs from naturally formed dsRNAs regulate transcripts in mouse oocytes. Nature 2008, 453:539-543.

7. Tam OH, Aravin AA, Stein P, Girard A, Murchison EP, Cheloufi S, Hodges E, Anger M, Sachidanandam R, Schultz RM, Hannon GJ: Pseudogene-derived small interfering RNAs regulate gene expression in mouse oocytes. Nature 2008, 453:534-538.

8. Ender C, Krek A, Friedlander MR, Beitzinger M, Weinmann L, Chen W Pfeffer S, Rajewsky N, Meister G: A human snoRNA with microRNA-like functions. Mol Cell 2008, 32:519-528.

9. Cole C, Sobala A, Lu C, Thatcher SR, Bowman A, Brown JW, Green PJ, Barton GJ, Hutvagner G: Filtering of deep sequencing data reveals the existence of abundant dicer-dependent small RNAs derived from tRNAs. RNA 2009, 15:2147-2160

10. Taft RJ, Glazov EA, Lassmann T, Hayashizaki Y, Carninci P, Mattick JS: Small RNAs derived from snoRNAs. RNA 2009, 15:1233-1240.

11. Lee YS, Shibata Y, Malhotra A, Dutta A: A novel class of small RNAs: tRNAderived RNA fragments (tRFs). Genes Dev 2009, 23:2639-2649.

12. Haussecker D, Huang $Y$, Lau A, Parameswaran P, Fire AZ, Kay MA: Human tRNA-derived small RNAs in the global regulation of RNA silencing. RNA 2010, 16:673-695.

13. Jinek M, Doudna JA: A three-dimensional view of the molecular machinery of RNA interference. Nature 2009, 457:405-412.

14. Czech B, Hannon GJ: Small RNA sorting: matchmaking for argonautes. Nat Rev Genet 2011, 12:19-31.

15. Ngo H, Tschudi C, Gull K, Ullu E: Double-stranded RNA induces mRNA degradation in Trypanosoma brucei. Proc Natl Acad Sci USA 1998, 95:14687-14692.

16. Shi H, Tschudi C, Ullu E: An unusual dicer-like1 protein fuels the RNA interference pathway in Trypanosoma brucei. RNA 2006, 12:2063-2072.

17. Patrick KL, Shi H, Kolev NG, Ersfeld K, Tschudi C, Ullu E: Distinct and overlapping roles for two dicer-like proteins in the RNA interference pathways of the ancient eukaryote Trypanosoma brucei. Proc Natl Acad Sci USA 2009, 106:17933-17938.

18. Shi H, Ullu E, Tschudi C: Function of the trypanosome argonaute 1 protein in RNA interference requires the N-terminal RGG domain and arginine 735 in the piwi domain. J Biol Chem 2004, 279:49889-49893.

19. Shi H, Chamond N, Djikeng A, Tschudi C, Ullu E: RNA interference in Trypanosoma brucei: the role of the amino-terminal RGG domain and the polyribosome association of argonaute1. J Biol Chem 2009, 284:36511-36520.

20. Barnes RL, Shi H, Kolev NG, Tschudi C, Ullu E: Comparative genomics reveals two novel RNAi factors in Trypanosoma brucei and provides insight into the core machinery. PLOS Pathog 2012, 8(5):e1002678. doi:10.1371/journal.ppat.1002678.

21. Djikeng A, Shi H, Tschudi C, Ullu E: RNA interference in Trypanosoma brucei: cloning of small interfering RNAs provides evidence for retroposon-derived 24-26-nucleotide RNAs. RNA 2001, 7:1522-1530.
22. Obado SO, Taylor MC, Wilkinson SR, Bromley EV, Kelly JM: Functional mapping of a trypanosome centromere by chromosome fragmentation identifies a 16-kb GC-rich transcriptional "strand-switch" domain as a major feature. Genome Res 2005, 15:36-43.

23. Shi H, Djikeng A, Tschudi C, Ullu E: Argonaute protein in the early divergent eukaryote Trypanosoma brucei: control of small interfering RNA accumulation and retroposon transcript abundance. Mol Cell Biol 2004, 24:420-427.

24. Berriman M, Ghedin E, Hertz-Fowler C, Blandin G, Renauld H, Bartholomeu DC, Lennard NJ, Caler E, Hamlin NE, Haas B, et al: The genome of the African trypanosome Trypanosoma brucei. Science 2005, 309: 416-422.

25. Ruben L, Egwuagu C, Patton CL: African trypanosomes contain calmodulin which is distinct from host calmodulin. Biochim Biophys Acta 1983, 758:104-113.

26. Hasan G, Turner MJ, Cordingley JS: Complete nucleotide sequence of an unusual mobile element from Trypanosoma brucei. Cell 1984, 37: 333-341.

27. Bringaud F, Biteau N, Melville SE, Hez S, El-Sayed NM, Leech V, Berriman M Neil Hall N, Donelson JE, Baltz T: A new, expressed multigene family containing a hot spot for insertion of retroelements is associated with polymorphic subtelomeric regions of Trypanosoma brucei. Eukaryot Cell 2002, 1:137-151.

28. Kimmel BE, Ole-MoiYoi OK, Young JR: Ingi, a 5.2-kb dispersed sequence element from Trypanosoma brucei that carries half of a smaller mobile element at either end and has homology with mammalian LINEs. Mol Cell Biol 1987, 7:1465-1475.

29. Murphy NB, Pays A, Tebabi P, Coquelet H, Guyaux M, Steinert M, Pays E: Trypanosoma brucei repeated element with unusual structural and transcriptional properties. J Mol Biol 1987, 195:855-871.

30. Pays $E$, Murphy NB: DNA-binding fingers encoded by a trypanosome retroposon. J Mol Biol 1987, 197:147-148.

31. Olivares M, Alonso C, Lopez MC: The open reading frame 1 of the L1TC retrotransposon of Trypanosoma cruzi codes for a protein with apurinicapyrimidinic nuclease activity. J Biol Chem 1997, 272:25224-25228.

32. Aksoy S, Lalor TM, Martin J, Van der Ploeg LH, Richards FF: Multiple copies of a retroposon interrupt spliced leader RNA genes in the African trypanosome, Trypanosoma gambiense. EMBO J 1987, 6:3819-3826.

33. Patrick KL, Luz PM, Ruan JP, Shi H, Ullu E, Tschudi C: Genomic rearrangements and transcriptional analysis of the spliced leaderassociated retrotransposon in RNA interference-deficient Trypanosoma brucei. Mol Microbiol 2008, 67:435-447.

34. White SA, Allshire RC: RNAi-mediated chromatin silencing in fission yeast. Curr Top Microbiol Immunol 2008, 320:157-183.

35. Benson G: Tandem repeats finder: a program to analyze DNA sequences. Nucleic Acids Res 1999, 27:573-580.

36. Kim VN, Han J, Siomi MC: Biogenesis of small RNAs in animals. Nat Rev Mol Cell Biol 2009, 10:126-139.

37. Okamura K, Balla S, Martin R, Liu N, Lai EC: Two distinct mechanisms generate endogenous siRNAs from bidirectional transcription in Drosophila melanogaster. Nat Struct Mol Biol 2008, 15:581-590.

38. Warburton PE, Giordano J, Cheung F, Gelfand Y, Benson G: Inverted repeat structure of the human genome: the $\mathrm{x}$-chromosome contains a preponderance of large, highly homologous inverted repeats that contain testes genes. Genome Res 2004, 14:1861-1869.

39. Altschul SF, Gish W, Miller W, Myers EW, Lipman DJ: Basic local alignment search tool. J Mol Biol 1990, 215:403-410.

40. Swaminathan J, Baxter EM, Corces VG: The role of histone H2Av variant replacement and histone $\mathrm{H} 4$ acetylation in the establishment of Drosophila heterochromatin. Genes Dev 2005, 19:65-76.

41. Dhillon N, Kamakaka RT: A histone variant, Htz1p, and a Sir1p-like protein, Esc2p, mediate silencing at HMR. Mol Cell 2000, 6:769-780.

42. Lowell JE, Kaiser F, Janzen CJ, Cross GA: Histone H2AZ dimerizes with a novel variant $\mathrm{H} 2 \mathrm{~B}$ and is enriched at repetitive DNA in Trypanosoma brucei. J Cell Sci 2005, 118:5721-5730.

43. Kato H, Goto DB, Martienssen RA, Urano T, Furukawa K, Murakami Y. RNA polymerase II is required for RNAi-dependent heterochromatin assembly. Science 2005, 309:467-469.

44. Gordenin DA, Lobachev KS, Degtyareva NP, Malkova AL, Perkins E, Resnick MA: Inverted DNA repeats: a source of eukaryotic genomic instability. Mol Cell Biol 1993, 13:5315-5322. 
45. Wen YZ, Zheng LL, Liao JY, Wang MH, Wei Y, Guo XM, Qu LH, Ayala FJ, Lun ZR: Pseudogene-derived small interference RNAs regulate gene expression in African Trypanosoma brucei. Proc Natl Acad Sci USA 2011 108:8345-8350

46. Kolev NG, Franklin JB, Carmi S, Shi H, Michaeli S, Tschudi C: The transcriptome of the human pathogen Trypanosoma brucei at singlenucleotide resolution. PLoS Pathog 2010, 6(5):e1001090. doi:1001010.1001371/journal.ppat.1001090.

47. Arhin GK, Shen S, Ullu E, Tschudi C: A PCR-based method for gene deletion and protein tagging in Trypanosoma brucei. Methods Mol Biol 2004, 270:277-286.

48. Goecks J, Nekrutenko A, Taylor J: Galaxy: a comprehensive approach for supporting accessible, reproducible, and transparent computational research in the life sciences. Genome Biol 2010, 11:R86.

49. Blankenberg D, Von Kuster G, Coraor N, Ananda G, Lazarus R, Mangan M, Nekrutenko A, Taylor J: Galaxy: a web-based genome analysis tool for experimentalists. Curr Protoc Mol Biol 2010, Chapter 19, Supplement 89: Unit 19.10.1-19.10.21

50. Giardine B, Riemer C, Hardison RC, Burhans R, Elnitski L, Shah P, Zhang Y, Blankenberg D, Albert I, Taylor J, et al: Galaxy: a platform for interactive large-scale genome analysis. Genome Res 2005, 15:1451-1455.

51. Langmead B, Trapnell C, Pop M, Salzberg SL: Ultrafast and memoryefficient alignment of short DNA sequences to the human genome. Genome Biol 2009, 10:R25.

doi:10.1186/1471-2164-13-427

Cite this article as: Tschudi et al:: Small interfering RNA-producing loci in the ancient parasitic eukaryote Trypanosoma brucei. BMC Genomics 2012 13:427.

\section{Submit your next manuscript to BioMed Central and take full advantage of:}

- Convenient online submission

- Thorough peer review

- No space constraints or color figure charges

- Immediate publication on acceptance

- Inclusion in PubMed, CAS, Scopus and Google Scholar

- Research which is freely available for redistribution 